\title{
TOWARD A NEW ABSTRACTION VOCABULARY FOR PROCESS MODELING
}

\author{
H T Goranson \\ Sirius-Beta, Virginia Beach, USA tedg@sirius-beta.com
}

\begin{abstract}
Process modeling for complex dynamic enterprises now requires several vexing types of meta and trans-modeling, for instance modeling processes that manage and change processes and modeling the modeling and metrification of processes. Current approaches are inadequate, cumbersome and impossible for average business users to understand. This paper reports on a newly forming project to tap popular vocabularies for sophisticated abstraction of manifold metastructures. A novelty is the focus on interactions in film. Another is the use of soft logics and group-theoretic metaphoric histories. Significant advances in modeling are expected, both in accessibility to users and in raw formal expressive power.
\end{abstract}

\section{INTRODUCTION}

Problems in system infrastructure are often a result of the history of their development, and virtual enterprise infrastructure is no different. The legacy of infrastructure for virtual enterprises is in first process modeling and then enterprise integration. "Process modeling" is the task of abstracting an explicit representation of a process in order to comprehend, engineer, manage and possibly execute it. The science of modeling is a matter of understanding abstraction, and is rather advanced. However, all mainstream process models work in a simple, two-layered abstraction universe; the two layers are the process and the model of that process (Hilbert, 1952).

Enterprise integration as a science is only a decade old. It grew out of the need to support an interactive community of models in rough conformance with the interaction of the real world of processes. The two-layered approach of process modeling was carried over as a legacy. The result is that processes were considered as atoms, modules or elements to be registered in some sort of framework. All practical integration frameworks use this two-layered approach (Vernadat, 1996). This was a satisfactory limitation as long as the goals of enterprise integration were along the lines of industrial engineering and addressed the actual work of the enterprise: assembly, logistics, workflow and such. Large, stable enterprises were the primary customer for this infrastructure and their productivity gains masked the limits of the two-layered abstraction. 
However, many solid studies (Goranson, 2003) indicated that the real problems with enterprise infrastructure concerned small enterprises and their affiliations, the fluid nature of affiliations and collaboration and (most importantly) business, legal, educational, financial processes along with various monitoring and certification processes. This became a core of the "virtual enterprise" infrastructure problem, made more attractive by the possibilities of conducting business using the resulting models over the internet.

At this point the two layered approach to abstraction becomes a severe restriction. This paper briefly outlines the several types of meta-abstraction needs that appear in the virtual enterprise problem, together with a few difficult additional factors. The need for a new approach to abstraction is noted and outlined. A promising, high risk approach is suggested, one around which new work is forming.

\section{VIRTUAL ENTERPRISE META-PROCESSES}

Virtual enterprises by definition do all the same work as static enterprises when it comes to processes associated with the actual product or service. These concern tasks that map directly to enterprise rewards and consist of such things as design, fabrication, logistics, sales and support. Ordinary, two-layer process models abstract these processes quite well. But there are a few types of processes that one could loosely characterize as meta-processes that also comprise the work of the enterprise. These are processes that work on other processes instead of the product or service. A simple example is a management process whose goals are the optimization of an assembly process. Such a meta-process actually works on the model of the assembly process rather than the process itself. In fact, the design of the assembly process model is often exclusively geared to support this meta-process.

All of a sudden, the abstraction requirements get very complicated. By actual survey, most enterprises of all kinds have more than half of all their processes in meta-processes of some type. This can range from $50 \%$ to $88 \%$ by actual count, up to $92 \%$ when measured by resources and costs (with a median of $71 \%$ ) (Goranson, 2002). By less scientific means, experience indicates that essentially all virtual enterprise failures and problems are a result of inadequate meta-process modeling and integration.

These meta-processes are of several types, briefly listed below.

\subsection{Semantic Meta-Processes}

All interesting enterprises use models created using different methods, degrees of formality and explicitness, different extents of correctness, fidelity and aptness, different granularities and often different ontologies. This is a simple fact in the real world, predating formal modeling and infrastructure frameworks but brought to new heights of complexity in the modern environment. The recognition of such problems and dealing with them are often embedded in other processes and when modeled get left out. Virtual enterprises, especially unplanned, opportunistic, first-time affiliations are severely restricted by a lack of ability to model these meta-processes. Mergers and acquisitions are similarly hampered. 


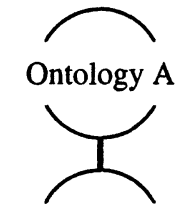

Model A

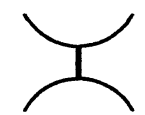

Real World A

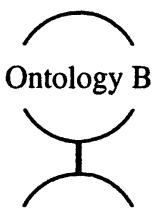

Model B

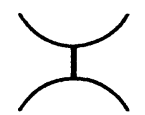

Real World B

Figure 1: Different Ontologies

The U. S. National Institute of Standards and Technology is beginning a novel program to address this requirement for meta-abstraction by attempting to define (and standardize) a meta-abstraction feature to capture the notion of "semantic distance."

\subsection{Monitoring Meta-Processes}

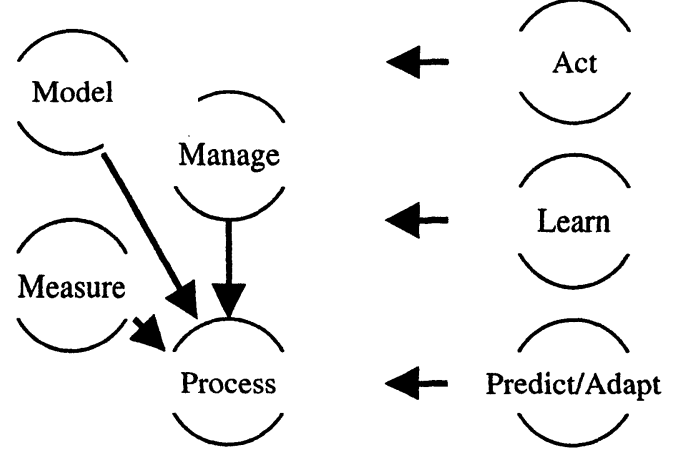

Figure 2: Simple, Direct Meta-Processes

As noted, there are a class of processes that work on processes. An obvious example are management and engineering processes. The features these processes address are the abstractions of the target models. But often those features themselves are the result of other processes; for example, management processes often work on abstractions resulting from a measurement process. In other words, one commonly finds a primary work process (say assembly) that is the target of a metrification process (say an activity-based costing accounting process) that produces its own meta-model, and the assembly management process works with abstractions from both the assembly and the accounting model. To complicate things, another manager (or even the same one) may be working with abstractions from other measurement processes: quality, throughput, workforce fatigue, environmental liabilities.

Worse, these meta-processes include some that are of fundamentally different types. The actual process of modeling is itself a modelable meta-process. The process of learning in the context of the stew of processes and their meta-processes involves a whole class of abstractions of a different type which one usually associates with adaptive systems and emergent behavior. Add to this meta-processes 
concerned with predicting and engineered adaptation and you have a highly complex lace of processes which are highly interdependent, have many overlays of metarelationship (sometimes both ways). Abstractions in many models are abstracted from abstractions in others.

\subsection{Multilevel Meta-Processes}

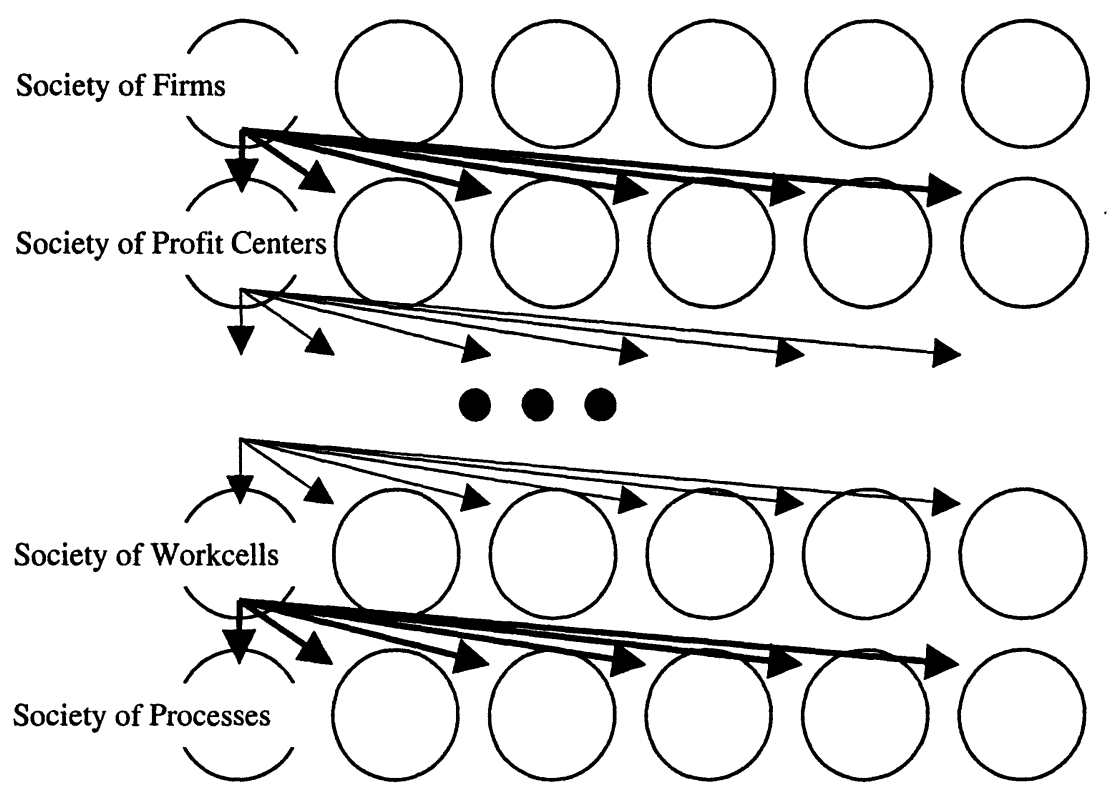

Figure 3: Meta-Domains

All that is bad enough, but there is another class of meta-relationships, with a different type of relationship between the features abstracted in a model and the meta-abstractions of a related model. This is the problem of enterprise levels (Stilman, 2000). A typical enterprise consists of several levels of collaborative processes at different levels of granularity. If the system is seen as a self-organizing system - as are many virtual enterprises - then some type of "bottom-up" emergent behavior will be at work. The "bottom" in this case is all of the processes and metaprocesses that form the fabric of the enterprise as previously noted. Most of those processes are contextualized at the level of granularity of the processes. That is, a collection of assembly processes self-optimize in the context of the community of other such processes, using a two-layer framework.

(Here, we use the term "layer" to mean degrees or meta-relationships of abstraction, and "level" to denote hierarchies in the enterprise. (Myers, 1995))

So we will have an emergent system where the basic unit is the process. Another level exists where workgroups or cells are the basic unit, or agent. Yet higher levels may exist of profit centers, firms and virtual enterprises. At each level, the unit acts selfishly to optimize the whole, at least nominally. Clearly, information moves from one level to the next (Hilfinger 1982). Just as clearly, the abstractions at different levels aren't the same (Barwise, 1990). An extreme example might be the 
prominence of group dynamics at the level of a five person team, and a similar prominence of corporate culture at the level of the firm.

There is some meta-abstraction that occurs here, only part of which is in the measurement of meta-processes previously noted. There is in fact, some layering of abstractions that mirrors the difference in collaborative semantics at different enterprise levels. This problem has an analogy in general studies of emergent behavior in natural systems where the semantics of interaction among elementary particles clearly influences chemical "collaboration" but uses different abstractions. The levels in that case step up through cells, organisms, and societies.

\subsection{Differing Causal Dynamics}

On top of it all, we have an even more vexing problem. A typical enterprise is a collection of often warring infrastructures: one or more each for different communities of interest; activity (including production), design, legal, accounting (what happened monetarily), and finance (what will happen monetarily) are major examples. Each of these has "home territory" in the enterprise. Most project their abstractions throughout the entire enterprise. Users who inhabit each of these semantic universes translate features in the others into their own using a manner of meta-abstraction different than noted above (with the exception of significant overlap between the accounting universe and the "measuring" meta-process of Section 2.2).

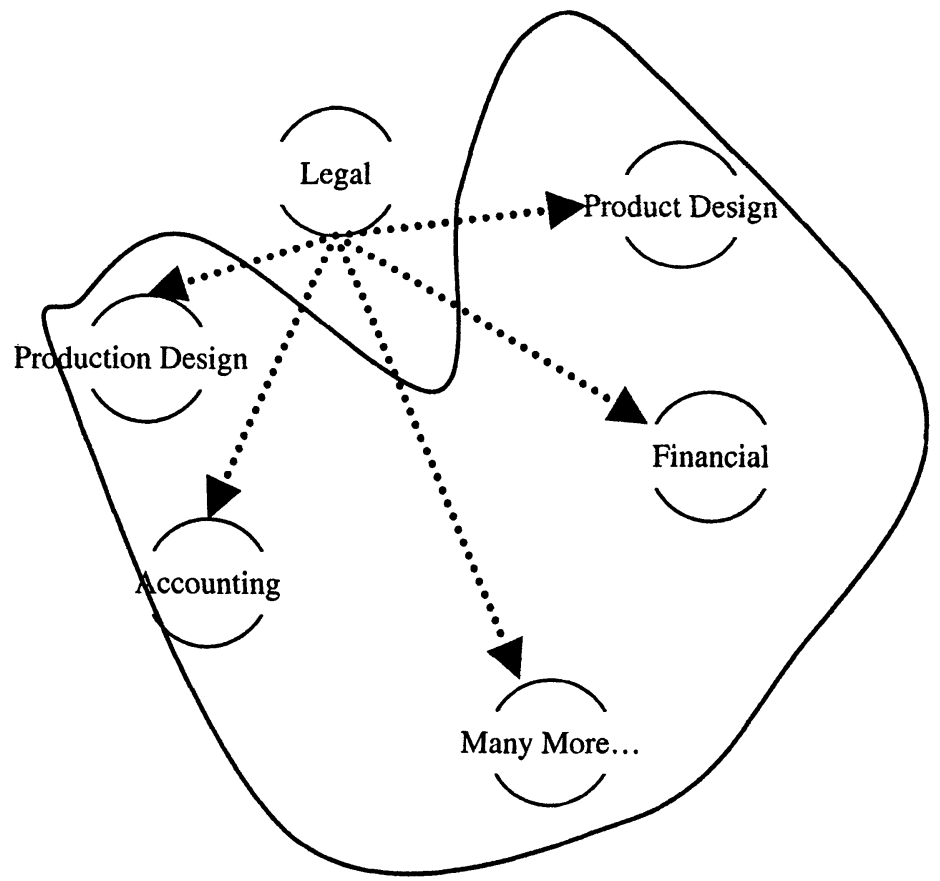

Figure 4: Differing World Views

Each world has different ontologies, but their difference goes beyond ontological mismatch, at least by the ordinary type of ontology (NRC, 1998). (The Process 
Specification Language is theoretically capable of spanning these differences but current sponsors are not interested in this problem.) Their world dynamics differ, sometimes in jarring ways. For instance, in the accounting world something exists only if it can be enumerated. In the legal world, "truth" is a relative matter having to do with admissibility rules. In the product world, features can be annotated to something physical, whereas features in the process are metaphysical. An average manager has numerous stories about some stupidity from one of these worlds caused by an inadequacy in their abstractions.

Each of these worlds situates the system context by different rules, even different logics. Any truly self-aware infrastructure must recognize and accommodate these multiply-directed concurrent meta-abstractions (Leyton, 1992).

\section{THE COMPLICATION OF "SOFTNESS"}

So we have several types of multi-layered abstraction, none of which are well satisfied with the two-layered default of process modeling and current integration frameworks. But there is another set of problems that complicates the puzzle: softness. We make the briefest mention here of the major problems:

No model will have its system context completely modeled (MacLaine, 1971). That is partly a matter of economy (why model the entire world to deal with one element of it), partly one of privacy (partners will not reveal all), partly one of ignorance (some elements and dynamics, especially social dynamics, are not feasibly modelable). Many of these systems have significant components, certainly concerning the larger economy, that are non-deterministic.

Another matter of "softness" is the intuitiveness of the models. In the process world, the process and its model are often conflated; in the product world, modelers have a very strong visual metaphor that binds the process and the model; in the financial world, the abstractions of the model have become real. In each of these worlds, at the two-layer abstraction level it is possible for a practitioner to be an expert in the discipline that the enterprise values and at the same time comprehend the mechanics of the model and its abstractions well enough. Not so with metamodels. So quite apart from the need for new meta-abstraction tools, and independent of the management of complexity, we have the problem of intuitive access.

Our group has led several workshops and studies on the problem of metaabstraction for the virtual enterprise (Goranson. 1999). The key problem seems clear: develop basic tools for meta-abstraction that are intuitive to the average high school graduate that also capture the notion of meta-layers and ambiguities as well as soft context. Actually, since these abstractions are usually the entryway for learning a discipline, they should be more intuitive and accessible than the "reality." This will empower the adoption of the methods in small enterprises and spawn new models of virtual enterprises, presumably resulting in greater productivity and higher quality of life.

But how? All existing research tends toward exotic methods that gives capabilities at the expense of being only useable by a few experts. 


\section{EMERGENT ABSTRACTION TECHNIQUES FOR INTERACTIVE EMERGENT SYSTEMS}

Our group has embarked on a high risk, high payoff strategy that might help with this agenda. Instead of working from the technical requirements and extracting new, probably esoteric capabilities, we are focusing on one type of abstraction methodology that has emerged in the general public. This general system of abstraction readily captures all the types of meta-abstraction noted above and then some. It is accessible to even illiterates, and non-native English speakers. It requires no mathematics to access, in fact is practiced by billions. In fact, many of the abstractions and meta-abstractions from this system have entered ordinary life as commonly used metaphors, some replacing of radically changing what was there.

We are talking about the collective methods used in films -- movies -- both within the story itself and implied in the manner of presenting the story. Some examples:

"Nurse Betty:" This is an actress (Renée Zellwiger), playing a housewife who thinks she is a character in a TeeVee program. She travels to Hollywood to join the TeeVee show. Once there, she pretends to be an actress in order to get the "part," which she does. During the production of the TeeVee show, she pretends to act. Meanwhile, another character is following her and observing the shifts between what is "real" and what representation. All the humor and plot devices depend on the mismatch among the abstractions.

"Adaptation" is a film about the writing of itself, and the chief character is the writer (taking the writer's real name). It is a meta-movie about a movie about a book about a magazine article about a botanical scam. Unlike "Nurse Betty" which had an actual plot, this film is all about its own self-reference, with all sorts of annotative fantasies commenting on itself and its own structure.

"Moulin Rouse" is an example of a different type, one in which someone in the film tells a story which leads to a story within which leads to another and another and they all run in parallel. The nesting is in opening a play, then a story told by a mystical crooner, then an absinthe vision, then a book, then a play within a play about the whole thing. Along the way is the notion of players as prostitutes, movies and songs as the same, gross anachronisms, all accompanied by that master of selfreference, Toulouse Latrec.

These were popular films, not art house projects. Collectively, they made about $\$ 200,000,000$. The meta-abstractions were easily registered with viewers; they did so. in fact, for pure enjoyment. These three examples are chosen somewhat at random. Our group has surveyed 1100 films so far and discovered about a third with this type of meta-abstraction, what we call "folding."

The notion is that quite apart from the content of the story, the form of the story in movies is an emergent system of abstraction methods (Kauffmann, 1994) that society uses to expand their capabilities for abstract reasoning. An essential assumption is that films are the vanguard in this respect. It follows that any metaabstraction not widely observed in films is not within the intuitive grasp of the target audience. 


\section{A RESEARCH AGENDA}

A new research project is forming. The goal is to empirically study a critical mass of films; discover emerging techniques of abstraction, formalize them in terms of themselves, in terms of the existing body of film theory and in modeling theory. Regarding the last, formal mathematical bases are sought, probably in extended situation logic and category and group theories. A goal is to supplement existing, cutting edge research in the modeling community such as the Process Specification Language and the Unified Enterprise Modeling Language.

The research plan is to involve an interdisciplinary group, adding new elements to those common in modeling, knowledge representation and enterprise integration. A key theme is self-describing interactivity (Gass, 1997). Media companies are expected to participate.

The project is in early stages, but a working taxonomy of meta-abstraction types and methods have been identified (Collier, 1998; Rosen, 2000), so the approach seems promising. Tentative decisions have been made as to appropriate mathematical tools. The project is intending to be self-documenting as it proceeds, using the same multimedia abstractions and models it discovers and describes.

\section{REFERENCES}

1. Barwise J, "Constraints, Channels and the Flow of Information". In Situation Theory and its Applications, Aczel P, Isreal D, Katagiri Y, Peters S eds. Palo Alto: Stanford University, 1990.

2. Collier J, "Order from Rhythmic Entrainment and the Origins of Levels through Dissipation". in Symmetry: Culture and Science. 1998; 9:2: 165-178.

3. Gass WH, Finding a Form. Ithica: Cornell University Press, 1996.

4. Goranson HT, The Agile Virtual Enterprise. Westport CT: Quorum Books, 1999.

5. Goranson HT, Cost Analyses and Survey of Meta=abstraction for Modeling. Norfolk: Classified DOD report, 2002.

6. Goranson HT, Chu B, Gruninger M, Ivezic N, Kulvantanyou S, Larbrou Y, Masouka R, Peng Y, Sheth A, Shorter D, "Ontologies as a New Cost Factor in Enterprise Integration". In Enterprise Inter and Intra 7. Organizational Integration, Kosanke K, Jochem R, Nell JG, Bas AO, eds. Berlin: Kluwer, 2003.

8. Hilbert D, Cohn-Vossen S, Geometry and the Imagination. New York: Chelsea, 1952.

9. Hilfinger PN, Abstraction Mechanisms and Language Design, Cambridge MA: MIT Press, 1982.

10. Leyton M, Symmetry Causality Mind. Cambridge MA: MIT Press, 1992.

11. Kauffmann S, Distinguishing Features. Baltimore: Johns Hopkins University Press, 1994.

12. MacLaine S, Categories for the Working Mathematician. New York: Springer-Verlag, 1971

13. Myers K, Konolige K, "Reasoning with Analogical Representations". In Diagrammatic Reasoning,

14. Glasgow J, Narayanan NH, Chandrasekaran B eds. Cambridge MA: MIT Press, 1995.

15. National Research Council, Visionary Manufacturing Challenges. Washington: National Academy Press, 1998.

16. Rosen R, "On Psychomimesis". In Essays on Life Itself. New York: Comulbia University Press, 2000.

17. Stilman B, Linguistic Geometry. Boston: Kluwer, 2002.

18. Vernadat FB, Enterprise Modeling and Integration. New York: Chapman \& Hall, 1996. 